\title{
Seasonal performance of a malaria rapid diagnosis test at community health clinics in a malaria-hyperendemic region of Burkina Faso
}

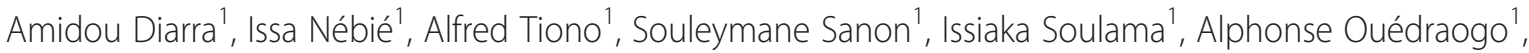 \\ Adama Gansané ${ }^{1}$, Jean B Yaro ${ }^{1}$, Espérance Ouédraogo ${ }^{1}$, Alfred S Traoré ${ }^{3}$ and Sodiomon B Sirima ${ }^{1,2^{*}}$
}

\begin{abstract}
Backgound: Treatment of confirmed malaria patients with Artemisinin-based Combination Therapy (ACT) at remote areas is the goal of many anti-malaria programs. Introduction of effective and affordable malaria Rapid Diagnosis Test (RDT) in remote areas could be an alternative tool for malaria case management. This study aimed to assess performance of the OptiMAL dipstick for rapid malaria diagnosis in children under five.

Methods: Malaria symptomatic and asymptomatic children were recruited in a passive manner in two community clinics (CCs). Malaria diagnosis by microscopy and RDT were performed. Performance of the tests was determined.

Results: RDT showed similar ability (61.2\%) to accurately diagnose malaria as microscopy (61.1\%). OptiMAL showed a high level of sensitivity and specificity, compared with microscopy, during both transmission seasons (high \& low), with a sensitivity of $92.9 \%$ vs. $74.9 \%$ and a specificity of $77.2 \%$ vs. $87.5 \%$.
\end{abstract}

Conclusion: By improving the performance of the test through accurate and continuous quality control of the device in the field, OptiMAL could be suitable for use at CCs for the management and control of malaria.

Keywords: Malaria diagnosis, Transmission season, RDT, OptiMAL

\section{Background}

Despite trends toward a reduction in reported malaria cases in many epidemiological settings, malaria remains a public health problem in the majority of countries in Sub-Saharan Africa. Throughout this region, because of the unavailability of microscopy as a tool, clinical symptoms are used to diagnosis malaria when rapid diagnosis is required in order to avoid the dramatic deterioration often seen in malariavulnerable patients such as children under five years old. Studies of fever cases in populations [1-3]from several malaria-endemic countries, however, have found wide variations in the proportion of fevers which can truly be attributed to malaria. These countries, including the Philippines, Sri Lanka, Thailand, Mali, Chad, Tanzania, and Kenya, have shown $40-80 \%$ rates of malaria over-

\footnotetext{
* Correspondence: s.sirima.cnlp@fasonet.bf

${ }^{1}$ Centre National de Recherche et de Formation sur le Paludisme, Ouagadougou, Burkina Faso

${ }^{2}$ Groupe de Recherche Action en Santé, Ouagadougou, Burkina Faso

Full list of author information is available at the end of the article
}

diagnosis, corresponding to potentially significant levels of associated economic loss. In Burkina Faso, a study of clinical examination-based malaria case definitions revealed that more than $50 \%$ of cases were not attributable to malaria alone [4]. Presumptive treatment of all fevers as malaria, previously a common practice, has therefore being questioned on economical grounds [5]. In addition, following the deployment of ACTs by the National Malaria Control Programs (NMCP); presumptive treatment is considered potentially dangerous as it might contribute to selecting for resistant Plasmodium falciparum strains. For these reasons, the new guidelines for malaria management recommend a mandatory laboratory test before beginning malaria treatment [6].

Microscopy has long been the method of choice for the diagnosis of many parasitic diseases, and until now it has also been the gold standard tool for malaria diagnosis. However, microscopy requires both technical skills and a power supply [7]. Solar microscopes used in the past are limited by a lack of reliable levels of sunlight, especially

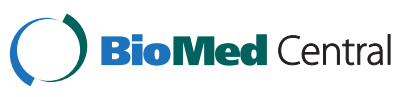


during the malaria high transmission season (rainy season), when most malaria cases are recorded. For efficient malaria case management at the rural level, an accurate, easy to use, and affordable malaria diagnostic tool is needed.

RDTs, introduced since the early 1990s for the detection of malaria parasites, exhibit high sensitivity and specificity compared with microscopy and Polymerase Chain Reaction [8-12]. Nevertheless, RDTs still have some limitations including the inability to detect mixed infections, all species of Plasmodium, infections at low concentrations of parasites, along with an inability to monitor response to therapy and false positives due to the presence of the malaria antigen even after successful treatment; however, their utilization in community clinics (CCs) where malaria transmission is hyper endemic and seasonal, could provide additional information for the NMCP, and would thereby serve to reduce the transmission, and consequently the burden of the disease.

Our epidemiological study aimed to assess the burden of malaria in children less than five years of age. We evaluated the sensitivity and specificity of OptiMAL compared with standard microscopy, in two CCs in the Saponé health district during malaria high and low transmission periods in 2007. OptiMAL, a dipstick test that has previously been performance tested by WHO [13] (DiaMed, Cressier, Switzerland), was used.

\section{Methods}

\section{Study area}

Study was conducted in the Saponé Health district in the province of Bazèga, located $50 \mathrm{~km}$ southwest of Ouagadougou, the capital city of Burkina Faso. The health district covers 79 villages and includes 14 CCs. The Centre National de Recherche et de Formation sur le Paludisme (CNRFP) is currently preparing this area for malaria vaccine trials. The climate of the area is characteristic of the Sudanese savannah, with a dry season from November to May (low transmission season), with a temperature range from $25^{\circ} \mathrm{C}$ to $30^{\circ} \mathrm{C}$ and a rainy season from June to October (high transmission season) with an average temperature range from $40^{\circ} \mathrm{C}$ to $42^{\circ} \mathrm{C}$ accompanied with higher humidity. Malaria transmission markedly occurs during the rainy season. Main vectors are Anopheles gambiae, and A. funestus. P. falciparum is the predominant malaria parasite, accounting for more than 95\% of infections in children under five years old [14].

Usage of insecticide-treated bednets in this area is very low (1.3\%), and indoor residual spraying is non-existent. Malaria control relies mainly on early diagnosis and adequate treatment of malaria episodes [14].

\section{Study population}

Main ethnic groups in this area are Mossi and Fulani. They are mostly subsistence farmers growing millet as well as raising domestic animals. Houses are made of mud, bricks and grass or corrugated iron roofs. The study population consisted mainly of children aged 0.5 to 5 years, residing in the study villages. Study children were drawn from an epidemiological study, which aimed to assess the malaria burden (mortality and morbidity) in the health district.

\section{Ethical clearance}

Ethical clearance was obtained from the ethics committee for biomedical research of the Ministry of Health of Burkina Faso.

\section{Study design and sample collection}

The purpose of the study was explained to the communities by the research team. The study was carried out between January 2007 and January 2008. Informed consent was obtained from all the participants' parents or legal guardians. The health staff of local CCs providing routine care were trained on the study procedures. Samples were collected in a passive manner as follows. Children attending the health facilities for care, presenting with fever (axillary temperature $\geq 37.5^{\circ} \mathrm{C}$ ) or a history of fever in the previous 24 hours were enrolled. Blood was taken by finger prick for blood smears and for the OptiMAL malaria RDT, performed according to the manufacturer's recommendations [15]. For microscopic diagnosis, blood smears were sent to the CNRFP laboratory.

To minimize observation bias, community clinic health workers performing RDT were blinded to the results of microscopic results. Technicians performing microscopic-based diagnosis were also blinded to the RDT results.

\section{Parasitological diagnosis by light microscope}

Blood films were air-dried, thin films fixed with methanol, and the slides were stained with Giemsa 6\%. Slides were read by experienced laboratory technicians who had been working mainly on malaria for many years in the CNRFP laboratory. The parasitological laboratory has been involved in the College of American Pathologist Proficiency (CAP) testing for 3 years, acheiving a good score, and most of the laboratory technicians were trained in the Malaria Diagnostic Centre of Excellence KEMRIWalter Reed Project in Kisumu (Kenya). Briefly, slides were read according to internal SOP as follow: 100 highpower fields (HPF) were examined, and the number of malaria parasites of each species and stage recorded. The number of parasites per microliter of blood was calculated, assuming 200 white blood cells per high power field and a fixed white cell count of $8000 / \mu$ l. A slide was considered negative if no parasites were found after $100 \mathrm{HPF}$ were examined. Each slide was read by two independent microscopists. In the event of a discrepancy between the 
two readers, in terms of species, presence or absence of malaria parasites, or if parasite densities differed by more than $30 \%$, the slide was re-examined by a third laboratory technician. Arithmetic mean of the two closest readings was used as the final value for parasite density. If there was no agreement after the third reading, the arithmetic mean of the three parasite densities was used.

\section{OptiMAL malaria rapid diagnostic test}

OptiMAL dipstick test is an immunochromatographic assay based on the detection of Plasmodium-specific lactate dehydrogenase (pLDH) in whole blood [16]. A positive result is defined as the presence of a colored (dark purple) band on the dipstick. The test includes an internal control band (top), a pan-Plasmodium-specific band (middle), and a $P$. falciparum-specific band (bottom). $\mathrm{pLDH}$ is produced only by living malaria parasites and has a short half-life in the blood $[17,18]$. RDT was supplied regularly to the CCs and kept at $+4^{\circ} \mathrm{C}$ to $+8^{\circ} \mathrm{C}$. All CCs staff members were thoroughly trained in the use of the test and interpretation of the test results before the beginning of the assessments.

One drop of whole blood was mixed with one drop of lysis buffer in well A. Lysis buffer disrupts red blood cells and releases $\mathrm{pLDH}$. The specimens were then allowed to migrate to the top of the pLDH strip. After ten minutes, strips were placed for washing in well B, containing 4 drops of buffer, which clears the haemoglobin from the strip and allows appearance of bands. Interpretation of test results was performed immediately. In the $\mathrm{pLDH}$ assay, there are two diagnostic zones of reaction containing different antibodies. The first diagnostic zone contains a monospecific antibody that recognizes only $P$. falciparum when present. The second diagnostic zone contains a pan-specific antibody immediately above the first zone. This monoclonal antibody recognizes the pLDH of all others species. A third reaction zone containing a pan-specific monoclonal antibody is present at the top of the test strip and serves as a positive control. The test is completed in 10-15 minutes.

\section{Data analysis}

Data double entry was done using Epi Info Software, and analysis performed using STATA, version 10 software (College Station, Texas 77845 USA) and Epi Info version 6 . Sensitivity and specificity of the test were estimated using microscopy as the gold standard (Table 1). Sensitivity, specificity, positive and negative predictive values were calculated as described elsewhere $[19,20]$.

The proportion of children presenting with fever or a reported history of fever with an RDT-positive test confirmed by microscopy was also calculated. Seasonal variation in the sensitivity and specificity of the test was estimated in relation to fever or history of fever. For each value, the $95 \%$ confidence interval $(95 \% \mathrm{CI})(\mathrm{P}=0.05)$ was calculated. A multivariate logistic regression model was
Table 1 Performance of the RDT compared to light microscopy

\begin{tabular}{lccc}
\hline RDT & \multicolumn{3}{c}{ Results of microscopy } \\
\cline { 2 - 4 } & Positive & Negative & Total \\
\hline Positive & 1768 & 235 & 2003 \\
Negative & 205 & 1011 & 1216 \\
Total & 1973 & 1246 & 3219 \\
\hline
\end{tabular}

performed to examine whether sensitivity and specificity of Optimal IT varied according to season age and parasite density.

\section{Results}

\section{Demographic and parasitological data}

A total of 1,242 children aged 0.5 to 5 years seeking care in two CCs were enrolled during the study period. During the study, 3, 219 visits were recorded in both clinics, with $62.4 \%$ of the visits registered during the malaria high transmission season and 37.6\% during the low transmission season. The majority of children attending the health facilities, $77.4 \%$, had axillary temperatures above $37.5^{\circ} \mathrm{C}$. The sex ratio, mean age, Plasmodium indexes, parasite densities during high and low transmission seasons are summarized in Table 2. Malaria-positive slides and malaria-positive RDTs were predominant during the malaria high transmission season, as compared with the low transmission season. Furthermore, Plasmodium indexes (P. falciparum, P. ovale and P. malariae) and densities of asexual stages were also high during the intense malaria transmission season. However, most of the RDT-positive samples represented P. falciparum nonspecific infections. Geometric mean of $P$. falciparum asexual stage density measured during the malaria high transmission season was threefold higher than that during the malaria low transmission season. P. falciparum was the main malaria species, with a prevalence of more than $94.2 \%$.

\section{Sensitivity and specificity of OptiMAL-IT during different} malaria transmission seasons in children with fever or history of fever reported within the previous 24 hours The overall sensitivity and specificity values of the OptiMAL-IT test during the study period were 89.6 (95\%CI 88.1-90.9) and 81.1\% (95\%CI 78.8-83.2), respectively, with negative and positive predictive values of 88.2\% (95\%CI 86.7-89.6) and 83.1\% (95\%CI 80.9-85.2), respectively (Tables 1 and 3). During the malaria high transmission season, sensitivities were $>90 \%$ in children with axillary temperatures of $37.5^{\circ} \mathrm{C}$ or more as well as in children with a history of fever. This dropped to less than $85 \%$ during the malaria low transmission season. The differences were statistically significant between the malaria high and low transmission seasons. In contrast, 
Table 2 Demographic and parasitological data

\begin{tabular}{|c|c|c|c|}
\hline & $\begin{array}{l}\text { Low transmission } \\
\text { season }(\mathrm{N}=1,210)\end{array}$ & $\begin{array}{l}\text { High transmission } \\
\text { season }(\mathrm{N}=2,009)\end{array}$ & $\begin{array}{c}\text { Overall } \\
(\mathrm{N}=3,219)\end{array}$ \\
\hline Sex ratio & 0.95 & 0.90 & 0.93 \\
\hline Mean age (years) & 1.3 & 1.2 & 1.3 \\
\hline Children with fever $\geq 37.5^{\circ} \mathrm{C}(\%)$ & 75.5 & 78.6 & 77.4 \\
\hline Children with history of fever (\%) & 24.5 & 21.4 & 22.6 \\
\hline Positive by microscopy (\%) & 41 & 73.3 & 61.1 \\
\hline Positive by RDT (\%) & 39.2 & 74.6 & 61.2 \\
\hline Positive by microscopy and negative by RDT (\%) & 10.7 & 5.2 & 7.3 \\
\hline Negative by microscopy and positive by RDT (\%) & 7.1 & 5.9 & 6.3 \\
\hline P. falciparum monospecific infection prevalence by microscopy (\%) & 37.8 & 71.1 & 58.6 \\
\hline RDT positive for $P$. falciparum monospecific infection prevalence (\%) & 28.9 & 66.3 & 52.2 \\
\hline P. falciparum gametocyte (alone) i prevalence by microscopy (\%) & 3.5 & 1.4 & 2.2 \\
\hline RDT positive for $P$. falciparum gametocyte alone (\%) & 0.8 & 0.8 & 0.8 \\
\hline P. malariae monospecific infection prevalence by microscopy (\%) & 1.1 & 0.4 & 0.7 \\
\hline RDT positive for $P$. malariae monospecific infection prevalence (\%) & 0.7 & 0.2 & 0.4 \\
\hline P. ovale monospecific infection prevalence by microscopy & 0.6 & 0.09 & 0.3 \\
\hline RDT positive for $P$. ovale monospecific prevalence (\%) & 0.1 & 0 & 0.2 \\
\hline Mixed Plasmodium infections* by microscopy & 3.1 & 2.1 & 2.5 \\
\hline RDT positive for mixed Plasmodium infections & 2.2 & 2 & 2.1 \\
\hline Geometric means of $P$. falciparum parasite density & $3921(3201-4804)$ & 14615(13187-16197) & \\
\hline
\end{tabular}

*Mixed infections $=P$. falciparum with $P$. malaria or $P$. ovale, or $P$. malariae with $P$. ovale.

specificity was high (>85\%) during the low transmission season, but decreased to less than $81 \%$ in both groups of children for the malaria high transmission season. The same patterns were observed for the predictive values (positive and negative).

Sensitivity of OptiMAL-IT at different levels of peripheral $P$. falciparum parasitemia during different malaria transmission seasons

Parasitemia was categorized into six groups in order to see the variation of the sensitivity of the RDT. Sensitivity of OptiMAL-IT increased for each of the six categories during both transmission seasons (Table 4). The highest sensitivities were recorded at parasite densities of $>10,000$ and performance dropped as parasite densities decreased.
Sensitivities of $>90 \%$ were reached at parasite densities of $>10,000$ during the low transmission season and $>1,000$ during the high transmission season.

\section{Sensitivity and specificity of OptiMAL-IT in different age} groups and during different malaria transmission seasons The sensitivity and specificity of the OptiMAL-IT has been shown to decrease with age within each malaria transmission season. The drop in both sensitivity and specificity is markedly pronounced in older children, as compared with younger infants (Table 5). During the malaria high transmission season, the sensitivity was $>90 \%$ in children younger than four years old and $<90 \%$ in all age groups at the malaria low transmission season. The specificities were above $80 \%$ in all age groups during the malaria low

Table 3 OptiMAL-IT performance during different malaria transmission seasons

\begin{tabular}{lcccc}
\hline RDT & $\begin{array}{c}\text { Low malaria } \\
\text { transmission season } \\
(\mathbf{9 5 \%} \mathbf{~ C l )}\end{array}$ & $\begin{array}{c}\text { High malaria } \\
\text { transmission season } \\
\mathbf{( 9 5 \% ~ C l )}\end{array}$ & $\mathbf{P}$ & $\begin{array}{c}\text { Overall } \\
\mathbf{( 9 5 \% ~ C l )}\end{array}$ \\
\hline Sensitivity (\%) & $74.9(70.9-78.5)$ & $92.9(91.5-94.1)$ & 0.001 & $88.2(86.7-89.6)$ \\
Specificity (\%) & $87.5(84.8-89.9)$ & $77.2(73.4-80.8)$ & 0.001 & $83.1(80.9-85.2)$ \\
PPV (\%) & $81.8(78.0-85.2)$ & $92(90.5-93.3)$ & 0.001 & $89.6(88.1-90.9)$ \\
NPV (\%) & $82.3(79.3-85.0)$ & $79.4(75.6-82.8)$ & 0.04 & $81.2(78.9-83.4)$ \\
\hline
\end{tabular}

$\mathrm{P}: \mathrm{P}$ value for comparison between low and high malaria transmission seasons. 
Table 4 Sensitivity of OptiMAL-IT at different levels of peripheral $P$. falciparum parasitemia during different malaria transmission seasons

\begin{tabular}{|c|c|c|c|c|c|}
\hline \multirow[t]{2}{*}{ Parasite threshold } & \multicolumn{2}{|c|}{ Low malaria transmission season } & \multicolumn{2}{|c|}{ High malaria transmission season } & \multirow[b]{2}{*}{$\mathbf{P}$} \\
\hline & $\mathbf{N}$ & Sensitivity $(95 \% \mathrm{Cl})$ & $\mathbf{N}$ & Sensitivity $(95 \% \mathrm{Cl})$ & \\
\hline$[0-100]$ & 29 & $10.2(2.1-27.3)$ & 37 & $45.9(29.4-63.0)$ & 0.001 \\
\hline$[100-500]$ & 76 & $40.7(29.6-52.6)$ & 91 & $67.0(56.3-76.5)$ & 0.001 \\
\hline$[500-1,000]$ & 41 & $56.0(39.7-71.5)$ & 66 & $71.2(58.7-81.6)$ & 0.11 \\
\hline$[1,000-5,000]$ & 109 & $82.5(74.1-89.1)$ & 184 & $90.2(84.9-94.0)$ & 0.05 \\
\hline$[5,000-10,000]$ & 37 & $83.7(67.9-93.8)$ & 114 & $98.2(93.8-99.7)$ & 0.002 \\
\hline$>10,000$ & 203 & $97.5(94.3-99.1)$ & 981 & $98.8(98.0-99.4)$ & 0.24 \\
\hline
\end{tabular}

P: P value for comparison between low and high malaria transmission seasons.

transmission season except in children $>4$ years. None of the five age bands had a specificity $\geq 80 \%$ for the malaria high transmission season.

Distribution of RDT false negative results by season, according to asexual parasite density

False negative rate varied similarly in both transmission seasons with more pronounced negative results with the low parasitemia (Table 6).

\section{Discussion}

Our results revealed that, in this rural area, children under five years old were carriers of malaria parasites at all time points of the malaria transmission season. More than half of them presenting with fever or a reported history of fever had malaria parasites in their blood, as detected either by microscopy (61.1\%) or RDT $(61.2 \%)$. Among these, $54.9 \%$ were RDT-positive, confirmed by microscopy. The same pattern was obtained with the OptiMAL dipstick assay, where $55.4 \%$ of all cases were diagnosed as positive by light microscopy vs. $48.8 \%$ by the OptiMAL test [21]. Thus, it appears that the performance of the OptiMAL is close to that of light microscope-based diagnosis under field conditions.

The study also showed that pLDH-based RDT performance varied seasonally in our study area. Malaria positivity rates determined by either light microscopy or RDT were high during the malaria high transmission season compared with low transmission season. Sensitivities were similarly affected by malaria transmission season, age and parasite density. Higher sensitivities were reported during the malaria high transmission season and children with high parasitemia were more likely to be positive by RDT than those with low parasitemia. This confirms previous findings from Madagascar, Kenya, and Uganda where the RDT positivities were found to be associated with high parasite load [22-26]. RDT positivity was also high in younger children, who are less immune compared with older children, confirming that sensitivity is affected by age-dependant immune status, as reported in a previous study [27].

Specificity of the RDT was not affected by age. However, it was affected by malaria transmission season; the number of false negative RDTs increased during the malaria high transmission season. These samples could have been falsely negative due to insufficient detection of low parasitemia, and pLDH levels in the samples were probably below the detection limit $(20 \mathrm{ng} / \mathrm{ml})$ of the RDT [25]. However, we cannot exclude that variability in the devices may have played a role in the performance of the test. False negative samples have been reported during both periods of transmission with higher parasitemia, and at least $40 \%$ of our subjects had parasitemia $>500 / \mu \mathrm{l}$. Though the underlying reason for this is unknown, similar findings have been reported in several earlier studies [22,28-31].

Clearly, the performance of the pLDH-based malaria RDT was greatly influenced by season. During the

Table 5 Sensitivity and specificity of OptiMAL-IT in different age groups and during different malaria transmission seasons

\begin{tabular}{|c|c|c|c|c|c|c|}
\hline \multirow{2}{*}{$\begin{array}{l}\text { Age group } \\
\text { (years) }\end{array}$} & \multicolumn{2}{|c|}{ Low malaria transmission season $(95 \% \mathrm{Cl})$} & \multicolumn{2}{|c|}{ High malaria transmission season $(95 \% \mathrm{Cl})$} & \multirow[b]{2}{*}{$\mathbf{P}$} & \multirow[b]{2}{*}{$P^{*}$} \\
\hline & Sensitivity (\%) & Specificity (\%) & Sensitivity (\%) & Specificity (\%) & & \\
\hline$[0-1]$ & $84.3(75.5-90.9)$ & $88.2(83.8-91.8)$ & $94.1(91.3-96.1)$ & $78.6(72.5-83.9)$ & 0.001 & 0.001 \\
\hline [1-2] & $82.6(74.0-89.4)$ & $89.2(83.0-93.6)$ & $93.5(90.5-95.8)$ & $76.6(68.6-83.4)$ & 0.001 & 0.001 \\
\hline$[2-3]$ & $76.6(68.6-83.4)$ & $89.7(82.7-94.5)$ & $94.3(91.3-96.5)$ & $74.7(64.5-83.2)$ & 0.001 & 0.001 \\
\hline$[3-4]$ & $68.2(58.4-77.0)$ & $88.6(79.4-94.6)$ & $92.5(87.9-95.7)$ & $80.0(66.2-89.9)$ & 0.001 & 0.01 \\
\hline$[4-5]$ & $58.1(46.0-69.4)$ & $79.4(67.8-88.2)$ & $84.8(77.9-90.2)$ & $77.4(58.9-90.4)$ & 0.001 & 0.6 \\
\hline
\end{tabular}

$\mathrm{P}: \mathrm{P}$ value for comparison of sensitivity between seasons; $\mathrm{P}$ : $\mathrm{P}$ value for comparison of specificity between malaria transmission seasons. 
Table 6 Malaria asexual parasite density and distribution of RDT false negative results, by season

\begin{tabular}{|c|c|c|c|c|}
\hline \multirow{2}{*}{ 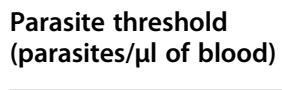 } & \multicolumn{2}{|c|}{ Low malaria transmission season $(\mathrm{N}=119)$} & \multicolumn{2}{|c|}{ High malaria transmission season $(\mathrm{N}=100$} \\
\hline & $\mathbf{N}$ & Frequency (\%) & $\mathbf{N}$ & Frequency (\%) \\
\hline$[0-100]$ & 26 & 21.8 & 20 & 20 \\
\hline$[100-500]$ & 45 & 37.8 & 30 & 30 \\
\hline$[500-1,000]$ & 18 & 15.1 & 19 & 19 \\
\hline$[1,000-5,000]$ & 19 & 15.9 & 18 & 18 \\
\hline$[5,000-10,000[$ & 11 & 09.2 & 13 & 13 \\
\hline
\end{tabular}

malaria high transmission season, the sensitivity of the test was $>90 \%$ in all age groups except children above four years of age. In comparison, during the malaria low transmission season, none of the age groups had sensitivities $>90 \%$. The RDT reached the level of $>90 \%$ sensitivity at parasitemia levels of $>1,000 / \mu \mathrm{l}$ during the malaria high transmission season, but it required a parasitemia of $>10,000 / \mu \mathrm{l}$ for the same sensitivity during the malaria low transmission season. It might be argued that the variability observed between the malaria transmissions seasons could have resulted from performance variability of the test used, especially as climatic conditions affect the stability of the devices. It has already been reported that stability is usually more problematic with pLDH-based tests than with HRP2-based tests [32].

Used in Papua New Guinea to monitor treatment outcomes, the OptiMAL dipstick showed a relatively low sensitivity (49.3\%) and specificity (59.1\%) [5]. The low sensitivity observed in our study may be due to the reduction in parasitemia from the treatment [22,23,33]. In a comparative study in India using OptiMAL,. Malik et al. obtained sensitivity and specificity values of $88.4 \%$ and $96.8 \%$, respectively, which are comparable to those obtained in our study [34]. In Burkina Faso, OptiMAL dipsticks used with 464 hospital patients recorded sensitivity and specificity of $98.70 \%$, and $96.25 \%$, respectively [35]. The relatively low RDT sensitivity and specificity levels observed in our study, compared with those obtained by Valéa using OptiMAL-IT, may be due partly to the fact that, in our study setting, children were actively followed up and referred to CCs for proper management of the malaria. This may have led to a drop in malaria parasite density and, consequently, a drop in the sensitivity and specificity of the test. However we cannot also exclude that self treatment by using traditional medicines and other conventional drugs, including antimalarials, before attending the community clinic may affect the sensitivity of the test. Other significant differences between the two studies include the sample size (large in our case) and also the design of the study; in the previous study, the tests were performed by laboratory technicians posted at the hospital, whereas in our study the tests were performed by trained community clinic health workers. The community health workers may not have processed the tests as accurately as laboratory Technicians because of the increasing workload brought by this new malaria diagnosis tool. In addition, there was no specific RDT monitoring program throughout the study period. Indeed, at the peripheral level, many factors such as work load, turnover of personnel, and control of the cold chain could have led to errors during test processing that may have affected the outcome of the RDT. Many studies have shown that job aids in the form of verbal or pictorial instructions, combined with training and supervision, enhance a health worker's ability to correctly perform specific tasks [36,37]. One caveat to be heeded when implementing an alternative measure, such as RDTs, to help health workers in remote areas to diagnose and manage malaria cases is that it must first be well accepted by the community health workers. A randomized trial conducted in Burkina Faso that aimed to determine the impact of the introduction of malaria RDT on clinical decisions, failed to show good compliance by health workers [38]. Preparation of appropriate instructions and training as well as monitoring of users' behavior and the cold chain is an essential part of rapid test implementation in remote areas.

\section{Conclusions}

These results, combined with other advantages of the OptiMAL test, such as the rapid availability of results (10-15 minutes) and relative simplicity compared with microscopy and other confirmatory tests done elsewhere, suggest that OptiMAL can be used by relatively inexperienced people to diagnose malaria infection in rural areas. However, seasonal variation should be considered when implementing an RDT-based malaria diagnostic program. Overall proportion of fever or reported history of fever associated with positive RDT and confirmed by microscopy indicates that at least half of outpatients presenting in our CCs are infected with malaria parasites at any given time point during the malaria transmission seasons. Despite the relatively low sensitivity of the test $(89.6 \%)$, the performance of the Optima IT test can be improved by putting in place an appropriate quality management system for the management of clinical malaria cases in remote areas. 


\section{Competing interests}

We declare that we have seen and approved the final version. We also declare that we have no conflict of interest in connection with this paper and state that the material has not already been published and will not be submitted for publication elsewhere so long as it is under consideration by the Journal of Parasites \& Vectors.

\section{Acknowledgments}

We express our gratitude to the study populations for their kind cooperation. The study was supported by the U.S. National Institutes of Health $(\mathrm{NIH})$ within the framework of DMID Protocol N06-20, Contract HHSN266200400016C

\section{Author details}

${ }^{1}$ Centre National de Recherche et de Formation sur le Paludisme, Ouagadougou, Burkina Faso. ${ }^{2}$ Groupe de Recherche Action en Santé, Ouagadougou, Burkina Faso. ${ }^{3}$ UFR Sciences de la Vie et de la Terre (SVT) Université de Ouagadougou, Ouagadougou, Burkina Faso.

\section{Authors' contributions}

All authors read and approved the final version of the manuscript.

Received: 26 September 2012 Accepted: 17 May 2012

Published: 30 May 2012

\section{References}

1. Dicko A, Mantel C, Kouriba B, Sagara I, Thera MA, Doumbia S, Diallo M, Poudiougou B, Diakite M, Doumbo OK: Season, fever prevalence and pyrogenic threshold for malaria disease definition in an endemic area of Mali. Trop Med Int Health 2005, 10(6):550-556.

2. Reyburn H, Ruanda J, Mwerinde O, Drakeley C: The contribution of microscopy to targeting antimalarial treatment in a low transmission area of Tanzania. Malar J 2006, 5:4.

3. Wongsrichanalai C, Barcus MJ, Muth S, Sutamihardja A, Wernsdorfer WH: A review of malaria diagnostic tools: microscopy and rapid diagnostic test (RDT). Am J Trop Med Hyg 2007, 77(6 Suppl):119-127.

4. Sirima BSI, Tiono AB, Konate AT: Données de base pour le suivi et l'évaluation de l'initiative "Faire reculer le Paludisme" et "Situation de la mise en oeuvre de l'initiative "Faire Reculer le Paludisme"dans les zones pilotes. Ouagadougou, Programme National de Lutte contre le Paludisme. 2003, :54-72.

5. Pfeiffer K, Some F, Muller O, Sie A, Kouyate B, Haefeli WE, Zoungrana A, Gustafsson LL, Tomson G, Sauerborn R: Clinical diagnosis of malaria and the risk of chloroquine self-medication in rural health centres in Burkina Faso. Trop Med Int Health 2008, 13(3):418-426.

6. Shillcutt S, Morel C, Goodman C, Coleman P, Bell D, Whitty CJ, Mills A: Cost-effectiveness of malaria diagnostic methods in sub-Saharan Africa in an era of combination therapy. Bull World Health Organ 2008, 86(2):101-110

7. Haditsch M: Quality and reliability of current malaria diagnostic methods. Travel Med Infect Dis 2004, 2(3-4):149-160.

8. Craig MH, Sharp BL: Comparative evaluation of four techniques for the diagnosis of Plasmodium falciparum infections. Trans $R$ Soc Trop Med Hyg 1997, 91(3):279-282.

9. Beadle C, Long GW, Weiss WR, McElroy PD, Maret SM, Oloo AJ, Hoffman SL: Diagnosis of malaria by detection of Plasmodium falciparum HRP-2 antigen with a rapid dipstick antigen-capture assay. Lancet 1994, 343(8897):564-568

10. Makler MT, Palmer CJ, Ager AL: A review of practical techniques for the diagnosis of malaria. Ann Trop Med Parasitol 1998, 92(4):419-433.

11. Mendiratta DK, Bhutada K, Narang R, Narang P: Evaluation of different methods for diagnosis of P. falciparum malaria. Indian J Med Microbiol 2006, 24(1):49-51.

12. Ashley EA, Touabi M, Ahrer M, Hutagalung R, Htun K, Luchavez J, Dureza C, Proux S, Leimanis M, Lwin MM, et al: Evaluation of three parasite lactate dehydrogenase-based rapid diagnostic tests for the diagnosis of falciparum and vivax malaria. Malar J 2009, 8:241.

13. WHO: Malaria Rapid Diagnosis Test Performance.2008. Round 1 (Results of WHO product testing of malaria RDT. Round 1 2008, 2008:P97.

14. Nebie I, Diarra A, Ouedraogo A, Soulama I, Bougouma EC, Tiono AB, Konate AT, Chilengi R, Theisen M, Dodoo D, et al: Humoral responses to Plasmodium falciparum blood-stage antigens and association with incidence of clinical malaria in children living in an area of seasonal malaria transmission in Burkina Faso, West Africa. Infect Immun 2008, 76(2):759-766.

15. Ratsimbasoa A, Randriamanantena A, Raherinjafy R, Rasoarilalao N, Menard D: Which malaria rapid test for Madagascar? Field and laboratory evaluation of three tests and expert microscopy of samples from suspected malaria patients in Madagascar. Am J Trop Med Hyg 2007, 76(3):481-485.

16. Iqbal J, Muneer A, Khalid N, Ahmed MA: Performance of the OptiMAL test for malaria diagnosis among suspected malaria patients at the rural health centers. Am J Trop Med Hyg 2003, 68 (5):624-628.

17. Piper R, Lebras J, Wentworth L, Hunt-Cooke A, Houze S, Chiodini P, Makler M: Immunocapture diagnostic assays for malaria using Plasmodium lactate dehydrogenase (pLDH). Am J Trop Med Hyg 1999, 60(1):109-118.

18. Fogg C, Twesigye R, Batwala V, Piola P, Nabasumba C, Kiguli J, Mutebi F, Hook C, Guillerm M, Moody A, et al: Assessment of three new parasite lactate dehydrogenase (pan-pLDH) tests for diagnosis of uncomplicated malaria. Trans R Soc Trop Med Hyg 2008, 102(1):25-31.

19. Iabal J, Sher A, Hira PR, Al-Owaish R: Comparison of the OptiMAL test with PCR for diagnosis of malaria in immigrants. J Clin Microbiol 1999, 37(11):3644-3646.

20. Mboera LE, Fanello Cl, Malima RC, Talbert A, Fogliati P, Bobbio F, Molteni F: Comparison of the Paracheck-Pf test with microscopy, for the confirmation of Plasmodium falciparum malaria in Tanzania. Ann Trop Med Parasitol 2006, 100(2):115-122.

21. Randrianasolo L, Tafangy PB, Raharimalala LA, Ratsimbasoa AC, Randriamanantena A, Randrianarivelojosia M: Rapid diagnostic test for malaria: preliminary study in Madagascar in 2003. Sante 2007, 17(2):69-73

22. Cooke AH, Chiodini PL, Doherty T, Moody AH, Ries J, Pinder M: Comparison of a parasite lactate dehydrogenase-based immunochromatographic antigen detection assay (OptiMAL) with microscopy for the detection of malaria parasites in human blood samples. Am J Trop Med Hyg 1999, 60(2):173-176.

23. Iqbal J, Khalid N, Hira PR: Comparison of two commercial assays with expert microscopy for confirmation of symptomatically diagnosed malaria. J Clin Microbiol 2002, 40(12):4675-4678.

24. Kyabayinze DJ, Tibenderana JK, Odong GW, Rwakimari JB, Counihan H: Operational accuracy and comparative persistent antigenicity of HRP2 rapid diagnostic tests for Plasmodium falciparum malaria in a hyperendemic region of Uganda. Malar J 2008, 7:221

25. Wanji S, Kimbi HK, Eyong JE, Tendongfor N, Ndamukong JL: Performance and usefulness of the Hexagon rapid diagnostic test in children with asymptomatic malaria living in the Mount Cameroon region. Malar J 2008, 7:89.

26. van Dijk DP, Gillet $P$, Vlieghe $E$, Cnops $L$, van Esbroeck M, Jacobs J: Evaluation of the Palutop +4 malaria rapid diagnostic test in a non-endemic setting. Malar J 2009, 8:293.

27. Fryauff DJ, Gomez-Saladin E, Purnomo, Sumawinata I, Sutamihardja MA, Tuti S, Subianto B, Richie TL: Comparative performance of the ParaSight $F$ test for detection of Plasmodium falciparum in malaria-immune and nonimmune populations in Irian Jaya, Indonesia. Bull World Health Organ 1997, 75(6):547-552.

28. Humar A, Ohrt C, Harrington MA, Pillai D, Kain KC: Parasight F test compared with the polymerase chain reaction and microscopy for the diagnosis of Plasmodium falciparum malaria in travelers. Am J Trop Med Hyg 1997, 56(1):44-48

29. Karbwang J, Tasanor O, Kanda T, Wattanagoon Y, Ibrahim M, Na-Bangchang K, Thanavibul A, Rooney W: ParaSight-F test for the detection of treatment failure in multidrug resistant Plasmodium falciparum malaria. Trans $R$ Soc Trop Med Hyg 1996, 90(5):513-515.

30. Palmer CJ, Lindo JF, Klaskala WI, Quesada JA, Kaminsky R, Baum MK, Ager AL: Evaluation of the OptiMAL test for rapid diagnosis of Plasmodium vivax and Plasmodium falciparum malaria. J Clin Microbiol 1998, 36(1):203-206.

31. Van den Ende J, Vervoort T, Van Gompel A, Lynen L: Evaluation of two tests based on the detection of histidine rich protein 2 for the diagnosis of imported Plasmodium falciparum malaria. Trans $R$ Soc Trop Med Hyg 1998, 92(3):285-288. 
32. Jorgensen P, Chanthap L, Rebueno A, Tsuyuoka R, Bell D: Malaria rapid diagnostic tests in tropical climates: the need for a cool chain. Am J Trop Med Hyg 2006, 74(5):750-754.

33. Jaeschke R, Guyatt GH, Sackett DL: Users' guides to the medical literature. III. How to use an article about a diagnostic test. B. What are the results and will they help me in caring for my patients? The Evidence-Based Medicine Working Group. JAMA 1994, 271(9):703-707.

34. Malik S, Khan S, Das A, Samantaray JC: Plasmodium lactate dehydrogenase assay to detect malarial parasites. Natl Med J India 2004, 17(5):237-239.

35. Valea I, Tinto H, Nikiema M, Yamuah L, Rouamba N, Drabo M, Guiguemde RT, d'Alessandro U: Performance of OptiMAL-IT compared to microscopy, for malaria detection in Burkina Faso. Trop Med Int Health 2009, 14(3):338-340

36. Harvey SA, Jennings L, Chinyama M, Masaninga F, Mulholland K, Bell DR: Improving community health worker use of malaria rapid diagnostic tests in Zambia: package instructions, job aid and job aid-plus-training. Malar I 2008, 7:160

37. Kim YM, Kols A, Martin A, Silva D, Rinehart W, Prammawat S, Johnson S, Church K: Promoting informed choice: evaluating a decision-making tool for family planning clients and providers in Mexico. Int Fam Plan Perspect 2005, 31(4):162-171.

38. Bisoffi Z, Sirima BS, Angheben A, Lodesani C, Gobbi F, Tinto H, Van den Ende J: Rapid malaria diagnostic tests vs. clinical management of malaria in rural Burkina Faso: safety and effect on clinical decisions. A randomized trial. Trop Med Int Health 2009, 14(5):491-498.

doi:10.1186/1756-3305-5-103

Cite this article as: Diarra et al:: Seasonal performance of a malaria rapid diagnosis test at community health clinics in a malaria-hyperendemic region of Burkina Faso. Parasites \& Vectors 2012 5:103.

\section{Submit your next manuscript to BioMed Central and take full advantage of:}

- Convenient online submission

- Thorough peer review

- No space constraints or color figure charges

- Immediate publication on acceptance

- Inclusion in PubMed, CAS, Scopus and Google Scholar

- Research which is freely available for redistribution 